\title{
Filamin B Enhances the Invasiveness of Cancer Cells into 3D Collagen Matrices
}

\author{
Yuta Iguchi $^{1}$, Seiichiro Ishihara ${ }^{1,2}$, Yoshimi Uchida ${ }^{1}$, Kaori Tajima ${ }^{1}$, Takeomi Mizutani ${ }^{1}$, \\ Kazushige Kawabata ${ }^{1}$, and Hisashi Haga ${ }^{1,2 *}$ \\ ${ }^{1}$ Faculty of Advanced Life Science, Hokkaido University, N10-W8, Kita-ku, Sapporo 060-0810, Japan, \\ ${ }^{2}$ Research Center for Cooperative Projects, Graduate School of Medicine, Hokkaido University, N15-W7, \\ Kita-ku, Sapporo 060-8638, Japan
}

\begin{abstract}
Numerous types of cancer cells migrate into extracellular tissues. This phenomenon is termed invasion, and is associated with poor prognosis in cancer patients. In this study, we demonstrated that filamin B (FLNb), an actin-binding protein, is highly expressed in cancer cell lines that exhibit high invasiveness, with a spindle morphology, into 3D collagen matrices. In addition, we determined that knockdown of FLNb in invasive cancer cells converts cell morphology from spindle-shaped, which is associated with high invasiveness, to roundshaped with low invasiveness. Furthermore, di-phosphorylation of myosin regulatory light chain (MRLC) and phosphorylation of focal adhesion kinase (FAK) are inhibited in FLNb-knockdown cancer cells. These results suggest that FLNb enhances invasion of cancer cells through phosphorylation of MRLC and FAK. Therefore, FLNb may be a new therapeutic target for invasive cancers.
\end{abstract}

Key words: cancer cells, filamin B, focal adhesion kinase, invasion, myosin regulatory light chain

\section{Introduction}

Some cancer cells are able to migrate into surrounding tissues. This phenomenon is called invasion and is a critical characteristic of cancer cells that are associated with poor prognosis in cancer patients. To migrate into tissues, cancer cells must reorganize actin filaments, a process regulated by actin binding proteins (Yamazaki et al., 2005).

Filamins are a group of actin binding proteins that consist of three isoforms: filamin A (FLNa), filamin B (FLNb), and filamin $\mathrm{C}$ (FLNc). They have some similar functions, including regulating the formation of the three-dimensional actin cytoskeleton network (Feng and Walsh, 2004; Stossel et al., 2001; Zhou et al., 2010). However, isoform-specific functions for filamin have been identified. For instance, FLNa plays a key regulatory role in apical extrusion (Kajita et al., 2014); FLNb contributes to angiogenesis by regulating the migration of endothelial cells (del Valle-Perez et al., 2010); FLNc is specifically expressed in cardiomyocytes and skeletal myocytes and promotes structural integrity (Fujita et al., 2012). In addition, FLNa is reported to enhance the invasive ability of human melanoma cells (Cunningham et al., 1992; Zhang et al., 2014). On the other

*To whom correspondence should be addressed: Hisashi Haga, Faculty of Advanced Life Science, Hokkaido University, N10-W8, Kita-ku, Sapporo 060-0810, Japan. hand, FLNa has been shown to prevent invasion and metastatic potential in human breast cancer cells ( $\mathrm{Xu}$ et al., 2010). Though the role of FLNa in regulating invasion has been characterized, the function of FLNb in this process is not well understood.

Contractile force is essential for cell migration. Cellular force is generated through actomyosin contractility, which is regulated by di-phosphorylation of myosin regulatory light chain (MRLC) (Mizutani et al., 2006). We have previously reported that the invasive ability of A549 lung cancer cells is regulated by di-phosphorylation of MRLC (Ishihara et al., 2013). In addition, FLNa promotes the phosphorylation of MRLC in human breast epithelial cells (Gehler et al., 2009). However, the role of FLNb in regulating MRLC phosphorylation it is not well studied.

Focal adhesions are adhesive structures that connect cells to their underlying substrate and consist of a large variety of proteins. Turnover of focal adhesions is important for cell migration (Bugide et al., 2014; Ezratty et al., 2005). Focal adhesion kinase (FAK) regulates the turnover of focal adhesions and is activated via phosphorylation at Y397, which subsequently promotes focal adhesion disassembly (Hamadi et al., 2005). FAK also contributes to invasion of cancer cells (Sulzmaier et al., 2014). Furthermore, a previous study indicated that FLNa inhibits turnover of focal adhesions (Xu et al., 2010). However, the role of FLNb in regulating focal adhesion turnover is not well understood. 
In this study, we demonstrate that FLNb is highly expressed in invasive cancer cells and contributes to their invasive ability. Furthermore, our data indicate that $\mathrm{FLNb}$ is critical for di-phosphorylation of MRLC and phosphorylation of FAK in invasive cancer cells.

\section{Materials and Methods}

\section{Cell culture}

A549 human lung adenocarcinoma cells and HT1080 human fibrosarcoma cells were purchased from American Type Culture Collection (ATCC; Manassas VA) and Riken Cell Bank (Tsukuba, Japan), respectively. Subclonal A549 cells (P-3 cells) and irradiation-tolerant P-3 cells (P-3IR cells) were generated as we previously reported (Ishihara et al., 2010). Cells were cultured using Dulbecco's Modified Eagle's Medium (DMEM; Sigma, St. Louis, MO) supplemented with 10\% fetal bovine serum (Bist-Tec; Equitech Bio Inc., Kerrville, TX, Biowest; Biowest, Nuaille, France) and 1\% antibiotic/antimycotic solution (Invitrogen, Carlsbad, CA). Cells were kept in a humidified incubator at $37^{\circ} \mathrm{C}$ with $5 \% \mathrm{CO}_{2}$.

\section{Reagents and antibodies}

Cellmatrix Type I-P (1.6 mg/mL; Nitta Gelatin Inc., Osaka, Japan) was used for preparing collagen gels. Primary antibodies used for the detection of filamins were anti-FLNa (Millipore, Billerica, MA, MAB1680) and anti-FLNb (Abcam, Cambridge, UK, ab97457) antibodies. Primary antibodies for western blotting included anti-di-phosphorylated myosin regulatory light chain (pp-MRLC, Cell Signaling Technology, Beverly, MA, \#3674), anti-total amount of myosin regulatory light chain (total-MRLC, Cell Signaling Technology, \#3672), anti- total amount of focal adhesion kinase (total-FAK, BD biosciences, San Jose, CA, 610087), anti- phosphorylated focal adhesion kinase at Y397 residue (FAK (pY397), BD biosciences, 611806), and anti-GAPDH antibody (Ambion, Foster City, CA, AM4300). Secondary antibodies of immunofluorescence staining included Alexa-Fluor 546 goat anti-mouse antibody (Molecular probes, Carlscad, CA, A-11003) and Alexa-Fluor 546 goat anti-rabbit antibody (Molecular probes, A-11010). Secondary antibodies for western blot analysis included HRP anti-mouse antibody (Bio-Rad, Hercules, CA, 70-6516) and HRP anti-rabbit antibody (Cell Signaling Technology, \#7074). Alexa-Fluor 488 phalloidin (Molecular probes, A-12379) was used for staining F-actin.

\section{siRNA transfection}

We generated siRNA duplexes using in vitro Transcription T7 kit (Takara Bio Inc., Otsu, Japan). The siRNA against FLNb targeted the sequence 5'-ATTTATCTTCGACTCTGATGG-3' (antisense sequence). FLNb siRNA or scrambled siRNA (as a negative control) were transfected into cells using Lipofectamine RNAiMAX
Reagent (Invirtogen). Knockdown of FLNb was confirmed three days after transfection using RT-PCR and/or western blotting.

\section{Collagen gel overlay method and time-lapse imaging}

The collagen gel solution $(150 \mu \mathrm{L})$ was added to a hand-made glass dish with a radius of $8 \mathrm{~mm}$, the bottom of which was made of commercial cover glass (Matsunami Glass Ind., Lid., Osaka, Japan). Either P-3IR or HT1080 cells $\left(3.0 \times 10^{3}\right.$ cells $)$ were seeded on the gel. Then, $24 \mathrm{~h}$ after seeding, $125 \mu \mathrm{L}$ of collagen solution was poured on top of the cells and incubated for $1 \mathrm{~h}$ in a humidified incubator at $37^{\circ} \mathrm{C}$ with $5 \% \mathrm{CO}_{2}$. The pouring of collagen provided the cells with a $3 \mathrm{D}$ culture environment. The dish was filled with growth medium and sealed with silicone grease. Time-lapse microscopy was performed by using a phase-contrast microscope (TE300, Nikon, Tokyo, Japan) with a $\times 10$ objective. During the experiment, the dish was kept at $37^{\circ} \mathrm{C}$ in an acrylic resin box. Time-lapse images were captured every $5 \mathrm{~min}$ using Image-Pro Software (Media Cybernetics Inc., Silver Spring, MD). The manual tracking plugin in Image $\mathrm{J}$ software (National Institutes of Health, Bethesda, MD) was used to track cell migration and analyze migration velocity. Student's $t$-test was used for statistical analysis.

\section{Immunofluorescence staining}

Cells were cultured for $24 \mathrm{~h}$ in a 3D collagen gel using the above described collagen gel overlay method. Cells were fixed using $4 \%$ formaldehyde in phosphate-buffered saline (PBS), permeabilized with $0.5 \%$ Triton $\mathrm{X}-100$ in PBS, and blocked using $0.5 \%$ of bovine serum albumin (Sigma) in PBS. The cells were incubated with primary antibodies (FLNa, 1:2000; FLNb, 1:250) in PBS for $1 \mathrm{~h}$ at room temperature. After three washes with PBS, the cells were incubated with secondary antibodies (Alexa-Fluor 546 goat anti-mouse antibody, 1:250, for FLNa staining; Alexa-Fluor 546 goat anti-rabbit antibody, 1:250, for FLNb staining) for $1 \mathrm{~h}$ at room temperature. After three washes with PBS, anti-bleaching reagent was added to the cells. Fluorescence images were obtained using confocal laser scanning microscopy (C1 confocal system; Nikon) with a $\times 60$ objective.

\section{Western blotting}

P-3 or P-3IR cells $\left(1.0 \times 10^{5}\right)$ were seeded onto plastic dishes (Corning, Tewksbury, MA) with a radius of $17.5 \mathrm{~mm}$ that were previously filled with $450 \mu \mathrm{L}$ of collagen gel. Twenty-four hours after seeding, $350 \mu \mathrm{L}$ of collagen gel solution was poured onto the top of the cells and incubated in a humidified incubator at $37^{\circ} \mathrm{C}$ with $5 \% \mathrm{CO}_{2}$. HT1080 cells $\left(1.0 \times 10^{5}\right)$ were seeded onto a plastic dish with a radius of $17.5 \mathrm{~mm}$ previously filled with $500 \mu \mathrm{L}$ of collagen gel. Twenty-four hours after seeding, $450 \mu \mathrm{L}$ of collagen gel solution was poured onto the cells and incubated in a humidified incubator at $37^{\circ} \mathrm{C}$ with $5 \% \mathrm{CO}_{2}$. Then, the cells were cultured for $24 \mathrm{~h}$. Cell extractions were prepared as we previously described (Ishihara et al., 2014). Cells were fixed with $10 \%$ trichloroacetic 
acid in PBS for $3 \mathrm{~min}$ on ice and then treated with $0.1 \%$ collagenase-L (Nitta Gelatin) in PBS for $90 \mathrm{~min}$ at $37^{\circ} \mathrm{C}$ with $5 \%$ $\mathrm{CO}_{2}$ to digest the collagen gel. Then, cells were isolated from the collagen solution using centrifugation. Cells were lysed using SDS sample buffer $(0.25 \mathrm{M}$ Tris- $\mathrm{HCl}, 5 \%$ of dithiothreitol, $2.3 \%$ of sodium dodecyl sulfate, $10 \%$ of glycerol, $0.01 \%$ of bromophenol blue, $\mathrm{pH}=6.8)$. Lysates were sonicated and boiled at $95^{\circ} \mathrm{C}$ for $5 \mathrm{~min}$. The lysates were separated using SDS-PAGE $(20 \mathrm{~mA}$ per gel, $70 \mathrm{~min}$ ). Further, $8.0 \%$ polyacrylamide gels were used in the detection of FLNa, FLNb, FAK (pY397), total-FAK, and GAPDH, whereas $12.5 \%$ polyacrylamide gels were used in the detection of pp-MRLC, total-MRLC, and GAPDH. After SDSPAGE, transfer to PDVF membranes (Millipore) was performed (92 mA per gel; $60 \mathrm{~min}$ for the detection of pp-MRLC, totalMRLC, and GAPDH; $150 \mathrm{~min}$ for the detection of FLNa, FLNb, FAK (pY397), total-FAK, and GAPDH). Next, membranes were blocked using $0.5 \%$ skim milk in TBS-Tween (for the detection of FAK (pY397), pp-MRLC, and GAPDH) or 5\% skim milk in TBSTween (for the detection of FLNa, FLNb, total-FAK, total-MRLC, and GAPDH) for $60 \mathrm{~min}$. The membranes were then incubated with primary antibodies (GAPDH, 1:50000-1:100000; FLNa, 1:500; FLNb, 1:200-1:500; FAK (pY397), 1:100-1:300; totalFAK, 1:100-1:300; pp-MRLC, 1:300; total-MRLC, 1:300) in TBS-Tween at $4{ }^{\circ} \mathrm{C}$ overnight. After three washes with TBSTween, the membranes were incubated with secondary antibodies (HRP anti-mouse antibody, 1:15000-1:100000, for the detection of FLNa, FAK (pY397), total-FAK, and GAPDH; HRP anti-rabbit antibody, 1:10000-1:50000, for the detection of FLNb, ppMRLC, and total-MRLC) in TBS-Tween for $1 \mathrm{~h}$ at room temperature. After three washes with TBS-Tween, signals were detected with Immobilon Western Chemiluminescent HRP substrate (Millipore). The band intensity was quantified using Image J software (National Institutes of Health). The levels of phosphorylated MRLC and FAK were determined using the ratio of the band intensity of pp-MRLC/total-MRLC and FAK (pY397)/total-FAK, respectively. Statistical analysis was performed using a Student's $t$-test.

\section{RT-PCR}

A plastic dish with a radius of $17.5 \mathrm{~mm}$ filled with $450 \mu \mathrm{L}$ of a collagen gel solution was prepared. P-3 or P-3IR cells $\left(1.0 \times 10^{5}\right)$ were seeded onto the dishes. Twenty-four hours after seeding, 350 $\mu \mathrm{L}$ of collagen gel solution was poured onto the cells and incubated in a humidified incubator at $37^{\circ} \mathrm{C}$ with $5 \% \mathrm{CO}_{2}$. $\mathrm{HT} 1080$ cells $\left(1.0 \times 10^{5}\right)$ were seeded onto a plastic dish without collagen gel and cultured for $48 \mathrm{~h}$; we were unable to obtain sufficient number of HT1080 cells in the collagen gel overlay for this experiment owing to low growth of the cells. RNA was isolated using TriPure Isolation reagent (Roche, Basel, Switzerland). Reverse transcription reaction was performed using a ReverTra Ace qPCR RT kit (Takara Bio Inc.). Then, polymerase chain reaction was performed using Taq DNA polymerase with ThermoPol buffer (TOYOBO, Osaka, Japan). We used following primers in the reactions: GAPDH, 5'-ACCACAGTCCATGCCATCAC-3' (upper) and 5'-
TCCACCACCCTGTTGCTGTA-3' (lower); FLNb, 5'-GGTGCTCAGCCAGAAGCGCA-3' (upper) and 5'-CAGGGCTCCCAGGGCTTTGC-3' (lower). The products were detected using electrophoresis with a $2 \%$ agarose gel.

\section{Results}

\section{FLNb expression is associated with invasiveness in cancer cells}

To investigate the relationship between filamin expression and invasiveness, we examined the protein expression levels of FLNa and FLNb in cancer cell lines with different invasive capacities. For these experiments, we utilized P-3 and P-3IR cells (Ishihara et al., 2010). Using a collagen gel overlay culture-method to analyze invasiveness in vitro, we previously reported that P-3IR cells, which have a spindleshaped morphology, are highly invasive than P-3 cells (Ishihara et al., 2010). Therefore, we compared the protein expression level of filamins in P-3 cells with P-3IR cells that were grown in collagen gel overlay culture. Using immunofluorescence staining and western blot analysis, we determined that the expression of FLNa was not significantly different between P-3 and P-3IR cells, whereas the expression of FLNb was higher in P-3IR cells than in P-3 cells (Fig. 1A, B and Fig. S1). Therefore, FLNb protein expression level is associated with a high invasive ability in human lung adenocarcinoma cells.

\section{FLNb is critical for invasiveness in irradiation-tolerant lung cancer cells}

Next, we investigated whether FLNb regulates invasiveness in P-3IR cells. We transfected P-3IR cells with scrambled siRNA, as a negative control, or siRNA targeting FLNb. Knockdown of FLNb was confirmed by RT-PCR and western blot analysis (Fig. 2A, B). We analyzed invasiveness of the cells in vitro by observation of morphology and migration using a collagen gel overlay culture-method. P-3IR cells transfected with scrambled siRNA invaded into the 3D collagen gel by generating protrusions (Fig. 2C). In this condition, cell migration was restricted to the horizontal direction (Movie S1). Therefore, we were able to analyze cell migration velocity using phase-contrast images. In contrast, FLNb knockdown cells exhibited low invasive ability and had a round-shaped morphology (Fig. 2C). Migration velocity in P-3IR cells transfected with scrambled siRNA was significantly greater than cells expressing siRNA targeting FLNb (Fig. 2D). These data indicate that FLNb contributes to the invasiveness of P-3IR cells into 3D collagen matrices. 


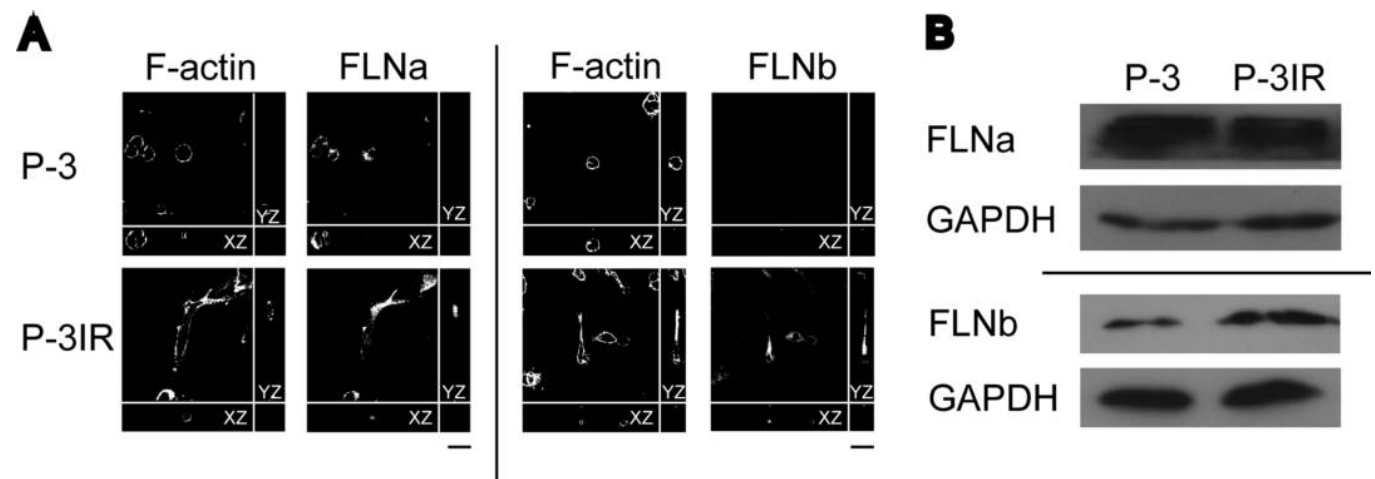

Fig. 1. Irradiation-tolerant lung cancer cells exhibit high protein expression levels of filamin B (FLNb). (A) Fluorescence images of F-actin, filamin A (FLNa), and FLNb in P-3 and P-3IR cells. XZ and YZ indicate cross-sectional views of the X-Z and Y-Z directions, respectively. Bar=25 $\mu \mathrm{m}$. (B) Western blot showing the protein expression of FLNa, FLNb, and GAPDH in P-3 and P-3IR cells.

A

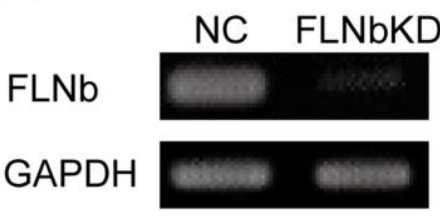

C

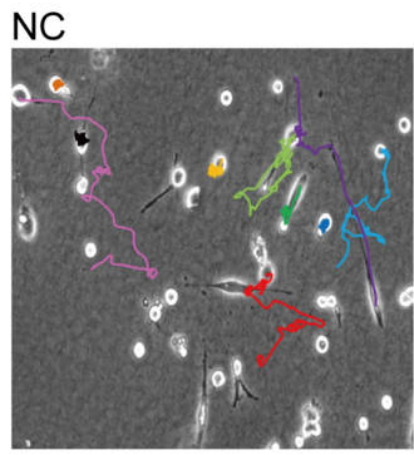

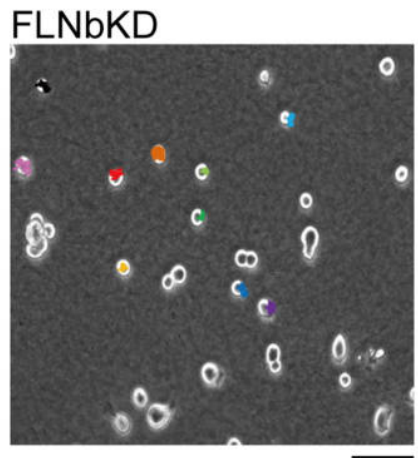

$\mathbf{B}$
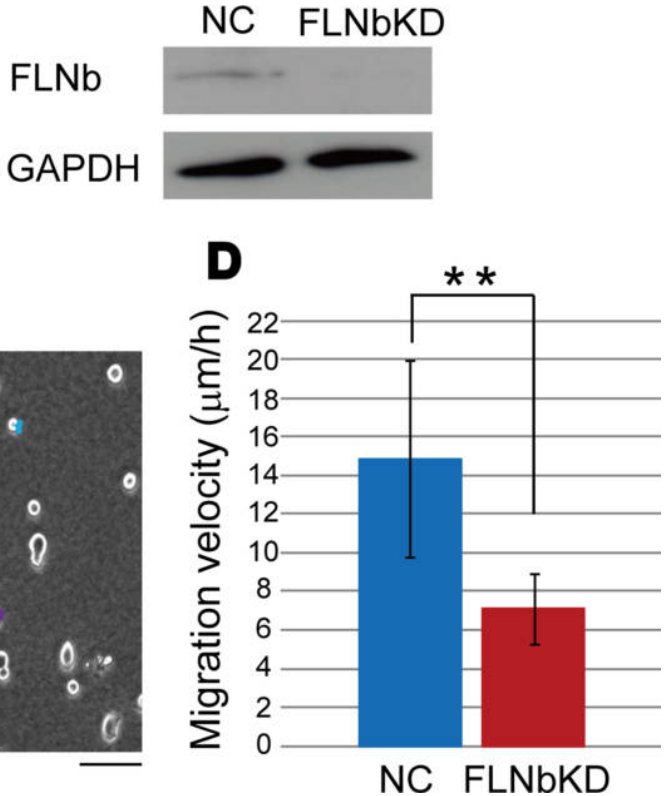

Fig. 2. Filamin B (FLNb) contributes to the invasiveness of irradiation-tolerant lung cancer cells. (A) mRNA expression of FLNb and GAPDH in P-3IR cells as detected by RT-PCR. P-3IR cells were transfected with scrambled siRNA as a negative control (NC) or siRNA targeting FLNb (FLNbKD). (B) Protein expression of FLNb in P-3IR cells detected by western blot analysis. (C) Time-lapse, phase-contrast images of P-3IR cells in a collagen gel. Colored lines in the images depict individual tracks of migrating P-3IR cells. Bar $=100 \mu \mathrm{m}$. (D) Statistical analysis of migration velocity in P-3IR cells. The mean values of 30 cells are shown with standard deviation (shown as the error bars) from 3 independent experiments. ${ }^{* *} \mathrm{p}<0.01$.

\section{FLNb plays an important role in the invasiveness of fibrosarcoma cells}

We examined the role of FLNb in regulating invasiveness in HT1080 human fibrosarcoma cells. HT1080 cells are known to invade into 3D collagen matrices while adopting spindle-shaped morphologies (Wolf et al., 2003), similarly to P-3IR cells. Knockdown of FLNb in HT1080 cells was confirmed using RT-PCR and western blot analysis (Fig.
3A, B). As seen with P-3IR cells, HT080 cells transfected with scrambled siRNA adopted an invasive phenotype, generating spindle-shaped protrusions into the $3 \mathrm{D}$ collagen gel (Fig. 3C). For HT1080 cells, migration was restricted to the horizontal direction for the collagen gel overlay (Movie S2). However, FLNb knockdown cells displayed a roundshaped morphology and had low invasive activity into the 3D collagen gel (Fig. 3C). The migration velocity of HT1080 cells transfected with scrambled siRNA was sig- 
A

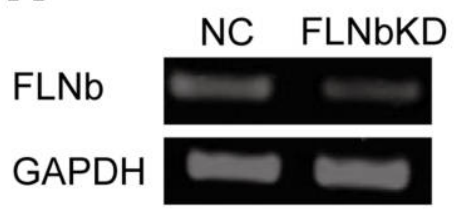

$\mathbf{B}$

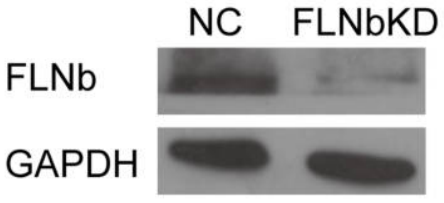

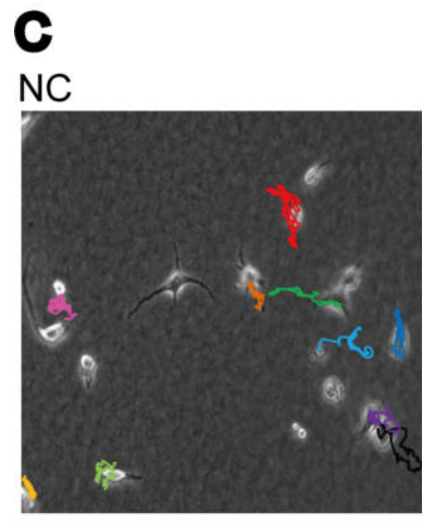
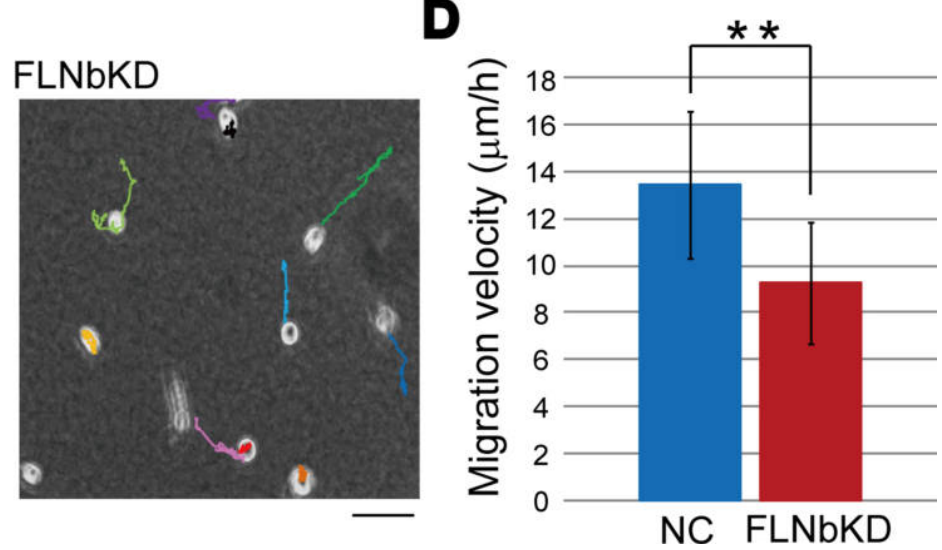

Fig. 3. Filamin B (FLNb) regulates invasiveness in fibrosarcoma cells. (A) mRNA expression of FLNb and GAPDH in HT1080 cells as detected by RTPCR. HT1080 cells were transfected with scrambled siRNA as a negative control (NC) or siRNA targeting FLNb (FLNbKD). (B) Protein expression of FLNb and GAPDH in HT1080 cells as detected by western blot analysis. (C) Time-lapse, phase-contrast images of HT1080 cells in a collagen gel. Colored lines indicate individual tracks of HT1080 cells. Bar=100 $\mu \mathrm{m}$. (D) Statistical analysis of migration velocity in HT1080 cells. The mean values of 30 cells are shown with standard deviation (shown as the error bars) from 3 independent experiments. ${ }^{* *} \mathrm{p}<0.01$.

nificantly faster than cells expressing siRNA targeting FLNb (Fig. 3D). Thus, FLNb plays an important role for the invasive phenotype of HT1080 cells, in addition to P-3IR cells.

\section{FLNb regulates di-phosphorylation of MRLC and phosphorylation of FAK}

We previously reported that di-phosphorylation of MRLC regulates invasiveness in P-3IR cells (Ishihara et al., 2013). Therefore, we assessed whether FLNb regulates diphosphorylation of MRLC in invasive cancer cells. Western blot analysis indicated that knockdown of FLNb in HT1080 cells reduced the level of di-phosphorylated MRLC (Fig. 4A, B). Therefore, FLNb regulates MRLC diphosphorylation in HT1080 cells.

In addition, we examined the role of FLNb in regulating FAK phosphorylation in HT1080 cells, which is known to contribute to invasion (Sulzmaier et al., 2014). We assessed the phosphorylation of residue Y397 in FAK, as this site is crucial for the activation of FAK and is associated with tumor progression (Sulzmaier et al., 2014). The level of phosphorylated FAK was significantly lower in FLNb knockdown HT1080 cells than in control cells (Fig. 4C, D). Therefore, phosphorylation of FAK at Y397 is regulated by FLNb expression in HT1080 cells.

\section{Discussion}

Our data indicate that FLNb contributes to invasiveness in cancer cells. We previously reported that P-3IR cells displayed an invasive phenotype with a spindle-shaped morphology, while P-3 cells exhibited low invasiveness and a round-shaped morphology in 3D collagen matrices (Ishihara et al., 2010, 2013). In this study, we demonstrated that P-3IR cells have a higher protein expression level of FLNb than P-3 cells. Furthermore, knockdown of FLNb induced a low-invasive phenotype and round morphology in P-3IR cells. Because P-3IR cells are radiation-tolerant cancer cells, we also investigated the contribution of FLNb to the invasiveness of non-irradiated HT1080 cells, which also indicates high invasiveness in a collagen gel. FLNb knockdown in HT1080 and P-3IR cells showed low invasiveness. Taken together, these data suggest that FLNb promotes invasive capability and a spindle-shaped morphology in cancer cells.

We also demonstrated that FLNb is a critical regulator of di-phosphorylation of MRLC and phosphorylation of Y397 in FAK in HT1080 cells. Di-phosphorylation of MRLC is important for generating contractile actomyosin forces (Mizutani et al., 2006). Furthermore, these contractile forces are crucial for the invasive ability of cancer cells (Friedl and Wolf, 2010). Thus, FLNb may enhance contrac- 


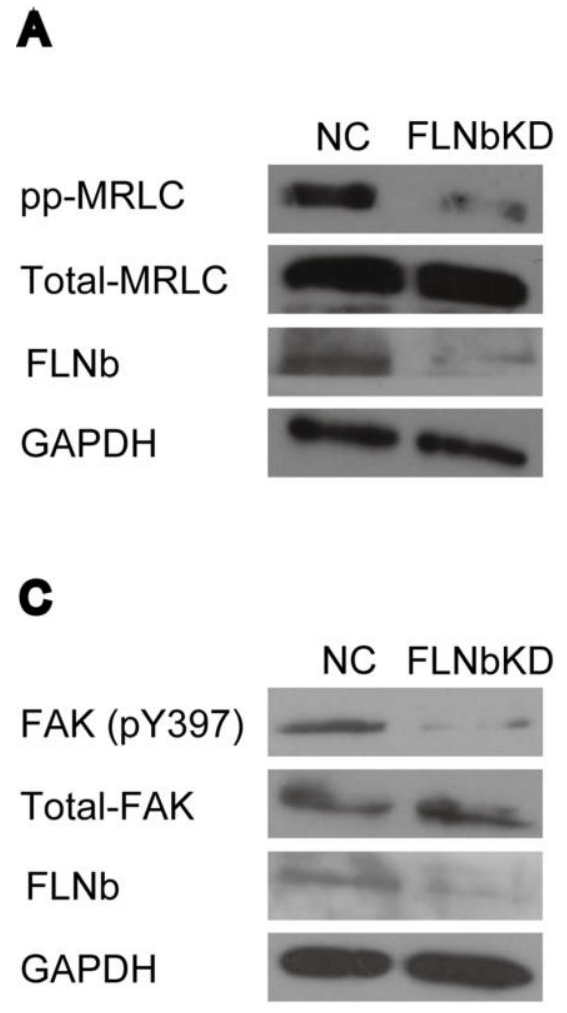

B

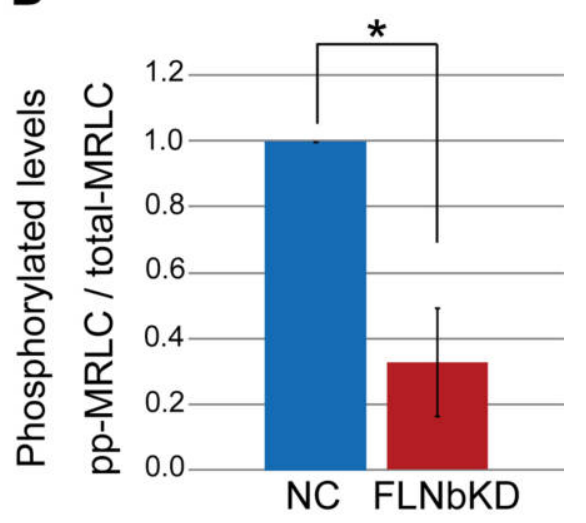

D

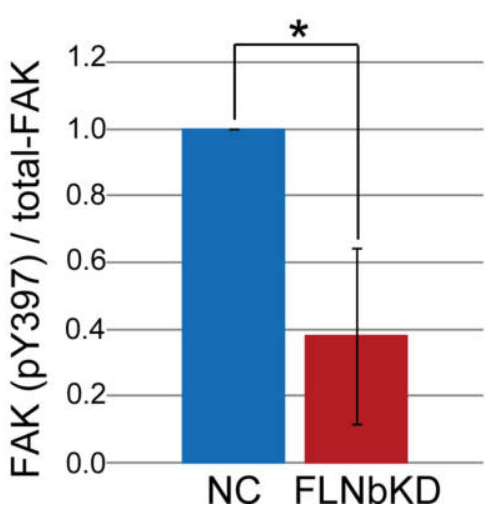

Fig. 4. Filamin B (FLNb) enhances phosphorylation of myosin regulatory light chain (MRLC) and focal adhesion kinase (FAK) in HT1080 cells. (A) Protein expression of di-phosphorylated MRLC (pp-MRLC), total amount of MRLC (total-MRLC), FLNb, and GAPDH in HT1080 cells as detected by western blot analysis is shown. (B) The relative phosphorylated level of MRLC (pp-MRLC/total-MRLC ratio) that was quantified from (A). The mean values are shown with standard deviation (shown as error bars) from 3 independent experiments. ${ }^{*} \mathrm{p}<0.05$. (C) Protein expression of phosphorylated FAK at Y397 residue (FAK (pY397)), total amount of FAK (total-FAK), FLNb, and GAPDH in HT1080 cells as detected by western blot analysis is shown. (D) The relative phosphorylated level of FAK (FAK (pY397)/total-FAK ratio) was calculated from (C). The mean values are shown with standard deviation (shown as error bars) from 3 independent experiments. ${ }^{*} \mathrm{p}<0.05$.

tile forces generated by actomyosin via regulation of diphosphorylation in MRLC. In addition, phosphorylation of FAK is reported to regulate focal adhesion turnover (Hamadi et al., 2005). Since turnover of focal adhesions is important for cell migration (Bugide et al., 2014), FLNb may promote invasion in cancer cells through FAKmediated regulation of focal adhesion turnover. Previous work has reported that di-phosphorylation of MRLC and phosphorylation of FAK at Y397 are both dependent on the RhoA-Rho kinase signaling cascade (Del Re et al., 2008; Mizutani et al., 2006). Therefore, FLNb could potentially regulate phosphorylation of MRLC and/or FAK by stimulating RhoA-Rho kinase signaling.

A recent report suggests that FLNb functions to repress malignant phenotypes of cancer cells, including invasiveness (Bandaru et al., 2014). These results are contradictory to the results presented here. This discrepancy could be due to differences in the experimental designs that were used. For example, Brandaru et al. used non-cancerous fibro- blasts and ovarian cancer cells. In this study, lung cancer cells and fibrosarcoma cells were used. Moreover, the previous study performed an invasion assay using a Matrigelcoated invasion chamber. Here, invasion was assessed by measuring cell migration into a $3 \mathrm{D}$ collagen matrix. It is possible that these methodological differences could account for the apparent observed discrepancies. In addition, FLNb may have different effects on cancer invasiveness, which may be dependent on cell type and extracellular environment.

Our data suggest that FLNb is a potential therapeutic target for invasive cancers, including lung cancer and fibrosarcoma. In this study, we demonstrated that downregulation of FLNb is sufficient to inhibit the invasive ability of lung carcinoma cells and fibrosarcoma cells. Therefore, inhibition of FLNb may improve the prognosis of patients with invasive cancer. 
Acknowledgments. The authors are grateful to Kosaku Kato for performing preliminary experiments. This study was supported by JSPS KAKENHI Grant Numbers 26430104, 26106702, 25127701, 24370069 to H.H., 26106704 to T.M., 25287106 to K.K., 26860964 to S.I. This study was also partly supported by Special Expenditures for "Reverse Translational Research from Advanced Medical Technology to Advanced Life Sciencc" to H.H. and S.I. granted by MEXT, Japan.

\section{References}

Bandaru, S., Zhou, A.X., Rouhi, P., Zhang, Y., Bergo, M.O., Cao, Y., and Akyurek, L.M. 2014. Targeting filamin B induces tumor growth and metastasis via enhanced activity of matrix metalloproteinase-9 and secretion of VEGF-A. Oncogenesis, 3: e119.

Bugide, S., David, D., Nair, A., Kannan, N., Samanthapudi, V.S., Prabhakar, J., and Manavathi, B. 2014. Hematopoietic PBX-interacting protein (HPIP) is over expressed in breast infiltrative ductal carcinoma and regulates cell adhesion and migration through modulation of focal adhesion dynamics. doi: 10.1038/onc.2014.38.

Cunningham, C.C., Gorlin, J.B., Kwiatkowski, D.J., Hartwig, J.H., Janmey, P.A., Byers, H.R., and Stossel, T.P. 1992. Actin-binding protein requirement for cortical stability and efficient locomotion. Science, 255: 325-327.

Del Re, D.P., Miyamoto, S., and Brown, J.H. 2008. Focal Adhesion Kinase as a RhoA-activable signaling scaffold mediating Akt activation and cardiomyocyte protection. J. Biol. Chem., 283: 35622-35629.

del Valle-Perez, B., Martinez, V.G., Lacasa-Salavert, C., Figueras, A., Shapiro, S.S., Takafuta, T., Casanovas, O., Capella, G., Ventura, F., and Vinals, F. 2010. Filamin B Plays a Key Role in Vascular Endothelial Growth Factor-induced Endothelial Cell motility through its interaction with Rac-1 and Vav-2. J. Biol. Chem., 285: 10748-10760.

Ezratty, E.J., Partridge, M.A., and Gundersen, G.G. 2005. Microtubuleinduced focal adhesion disassembly is mediated by dynamin and focal adhesion kinase. Nat. Cell Biol., 7: 581-U515.

Feng, Y.Y. and Walsh, C.A. 2004. The many faces of filamin: A versatile molecular scaffold for cell motility and signalling. Nat. Cell Biol., 6: 1034-1038.

Friedl, P. and Wolf, K. 2010. Plasticity of cell migration: a multiscale tuning model. J. Cell Biol., 188: 11-19.

Fujita, M., Mitsuhashi, H., Isogai, S., Nakata, T., Kawakami, A., Nonaka, I., Noguchi, S., Hayashi, Y.K., Nishino, I., and Kudo, A. 2012. Filamin $\mathrm{C}$ plays an essential role in the maintenance of the structural integrity of cardiac and skeletal muscles, revealed by the medaka mutant zacro. Dev. Biol., 361: 79-89.

Gehler, S., Baldassarre, M., Lad, Y., Leight, J.L., Wozniak, M.A., Riching, K.M., Eliceiri, K.W., Weaver, V.M., Calderwood, D.A., and Keely, P.J. 2009. Filamin A-beta 1 Integrin Complex Tunes Epithelial Cell Response to Matrix Tension. Mol. Biol. Cell, 20: 3224-3238.

Hamadi, A., Bouali, M., Dontenwill, M., Stoeckel, H., Takeda, K., and Ronde, P. 2005. Regulation of focal adhesion dynamics and disassembly by phosphorylation of FAK at tyrosine 397. J. Cell Sci., 118: 44154425.
Ishihara, S., Haga, H., Yasuda, M., Mizutani, T., Kawabata, K., Shirato, H., and Nishioka, T. 2010. Integrin beta 1-dependent invasive migration of irradiation-tolerant human lung adenocarcinoma cells in 3D collagen matrix. Biochem. Biophys. Res. Commun., 396: 651-655.

Ishihara, S., Yasuda, M., Nishioka, T., Mizutani, T., Kawabata, K., Shirato, H., and Haga, H. 2013. Irradiation-tolerant lung cancer cells acquire invasive ability dependent on dephosphorylation of the myosin regulatory light chain. Febs Lett., 587: 732-736.

Ishihara, S., Mizutani, T., Kawabata, K., and Haga, H. 2014. An improved method for western blotting when extracting proteins from mammalian cells cultured on a collagen gel under serum-free conditions. Cytotechnology, doi: 10.1007/s10616-014-9766-4.

Kajita, M., Sugimura, K., Ohoka, A., Burden, J., Suganuma, H., Ikegawa, M., Shimada, T., Kitamura, T., Shindoh, M., Ishikawa, S., Yamamoto, S., Saitoh, S., Yako, Y., Takahashi, R., Okajima, T., Kikuta, J., Maijima, Y., Ishii, M., Tada, M., and Fujita, Y. 2014. Filamin acts as a key regulator in epithelial defence against transformed cells. Nat. Commun., $\mathbf{5}$ : 13.

Mizutani, T., Haga, H., Koyama, Y., Takahashi, M., and Kawabata, K. 2006. Diphosphorylation of the myosin regulatory light chain enhances the tension acting on stress fibers in fibroblasts. J. Cell. Physiol., 209: 726-731.

Stossel, T.P., Condeelis, J., Cooley, L., Hartwig, J.H., Noegel, A., Schleicher, M., and Shapiro, S.S. 2001. Filamins as integrators of cell mechanics and signalling. Nat. Rev. Mol. Cell Biol., 2: 138-145.

Sulzmaier, F.J., Jean, C., and Schlaepfer, D.D. 2014. FAK in cancer: mechanistic findings and clinical applications. Nat. Rev. Cancer, 14: 598-610.

Wolf, K., Mazo, I., Leung, H., Engelke, K., von Andrian, U.H., Deryugina, E.I., Strongin, A.Y., Brocker, E.B., and Friedl, P. 2003. Compensation mechanism in tumor cell migration: mesenchymalamoeboid transition after blocking of pericellular proteolysis. J. Cell Biol., 160: 267-277.

Xu, Y.J., Bismar, T.A., Su, J., Xu, B., Kristiansen, G., Varga, Z., Teng, L.H., Ingber, D.E., Mammoto, A., Kumar, R., and Alaoui-Jamali, M.A. 2010. Filamin A regulates focal adhesion disassembly and suppresses breast cancer cell migration and invasion. J. Exp. Med., 207: 24212437.

Yamazaki, D., Kurisu,S., and Takenawa, T. 2005. Regulation of cancer cell motility through actin reorganization. Cancer Sci., 96: 379-386.

Zhang, K., Zhu, T.N., Gao, D.M., Zhang, Y.M., Zhao, Q.L., Liu, S., Su, T.Y., Bernier, M., and Zhao, R.J. 2014. Filamin A expression correlates with proliferation and invasive properties of human metastatic melanoma tumors: implications for survival in patients. J. Cancer Res. Clin., 140: $1913-1926$.

Zhou, A.X., Hartwig, J.H., and Akyurek, L.M. 2010. Filamins in cell signaling, transcription and organ development. Trends Cell Biol., 20: 113123.

(Received for publication, March 10, 2015, accepted, April 22, 2015 and published online, April 29, 2015) 